\title{
Estimation and Testing Procedures for the Reliability Functions of a Family of Lifetime Distributions Based on Records
}

\author{
Ajit Chaturvedi and Ananya Malhotra \\ Department of Statistics, University of Delhi, Delhi - 110007, INDIA
}

\begin{abstract}
A family of lifetime distributions is considered. Two measures of reliability are considered, $R(t)=P(X>t)$ and $P=P(X>Y)$. Point estimation and testing procedures are developed for $R(t)$ and $P$ based on records. Two types of point estimators are developed uniformly minimum variance unbiased estimators (UMVUES) and maximum likelihood estimators (MLES). A comparative study of different methods of estimation is done through simulation studies. Testing procedures are developed for the hypothesis related to different parametric functions.
\end{abstract}

Keywords and Phrases: family of lifetime distributions; point estimation; testing procedures; records; simulation studies.

\section{Introduction}

The reliability function $R(t)$ is defined as the probability of failure-free operation until time $t$. Thus, if the random variable $(r v) X$ denotes the lifetime of an item or a system, then $R(t)=P(X>t)$. Another measure of reliability under stress-strength setup is the probability $P=P(X>Y)$, which represents the reliability of an item or a system of random strength $X$ subject to random stress $Y$. In engineering, stress is a solid body (liquids do not admit engineering stress) arises due to applied loads and is defined as "the force per unit area that one part of the body exerts on adjacent parts". Psychological stress is another type of stress. A lot of work has been done in the literature for the point estimation and testing of $R(t)$ and $P$. For example, Pugh (1963), Basu (1964), Bartholomew (1957, 1963), Tong (1974, 1975), Johnson (1975), Kelley, Kelley and Schucany (1976), Sathe and Shah (1981), Chao (1982), Chaturvedi and Surinder (1999) developed inferential procedures for $R(t)$ and $P$ for exponential distribution. Constantine, Karson and Tse (1986) derived UMVUE and MLE for $P$ associated with gamma distribution. Awad and Gharraf (1986) estimated $P$ for Burr distribution. For estimation of $R(t)$ corresponding to Maxwell and generalized Maxwell distributions, one may refer to Tyagi and Bhattacharya (1981) and Chaturvedi and Rani (1998), respectively. Inferences have been drawn for $R(t)$ and $P$ for some families of lifetime distributions by Chaturvedi and Rani (1997), Chaturvedi and Tomer (2003), Chaturvedi and Singh (2006, 2008) and Chaturvedi and Kumari (2015). Chaturvedi and Tomer (2002) derived UMVUE for $R(t)$ and $P$ for negative binomial distribution. For exponentiated Weibull and Lomax distributions, the inferential procedures are available in Chaturvedi and Pathak (2012, 2013, 2014).

Chandler (1952) introduced the concept of record values. Based on records, inferential procedures for the parameters of different distributins have been developed by Glick (1978), Nagaraja (1988a,1988b), Balakrishan, Ahsanullah and Chan (1995), Arnold, Balakrishan and Nagaraja (1992), Habibi Rad, Arghami and Ahmadi (2006), Arashi and Emadi (2008), Razmkhah and Ahmadi (2011), Arabi Belaghi, Arashi and Tabatabaey (2015) and others. 
No one can resist being interested in record values. The hottest day ever, the longest winning streak in professional basketball, the lowest stock market figure, these we cannot resist. To the best of the knowledge of authors, no inferential procedures are available in the literature for the estimation and testing of reliability functions based on records. The records take values in life-testing experiments also.

The purpose of this paper is many-fold. We consider a family of lifetime distributions, which covers as many as fourteen distributions as its specific cases. We develop point estimation and testing procedures based on records. As far as point estimation is concerned, we derive UMVUES and MLES. A new technique of obtaining these estimators is developed, in which first of all the estimators of powers of parameter are obtained. These estimators are used to obtain estimators of $R(t)$. Using the derivatives of the estimators of $R(t)$, the estimators of sampled probability density function $(p d f)$, at a specified point, are obtained which are subsequently used to obtain estimators of $P$. The estimators of $P$ are derived for the cases when $X$ and $Y$ belong to the same and different families of distributions. Test procedures are developed for different hypotheses.

In Section 2, we give the family of lifetime distributions. In Section 3 and Section 4, respectively, we develop point estimation procedures and testing procedures. Finally, in Section 5, we present numerical findings.

\section{The Family of Lifetime Distributions}

Let the $r v X$ follow the distribution having the $p d f$

$f(x ; a, \lambda, \underline{\theta})=\frac{G^{\prime}(x ; a, \underline{\theta})}{\lambda} \exp \left(-\frac{G(x ; a, \underline{\theta})}{\lambda}\right) ; x>a \geq 0, \lambda>0$.

Here, $G(x ; a, \underline{\theta})$ is a function of $x$ and may also depend on the parameters $a$ and $\underline{\theta}$. $\underline{\theta}$ may be vector valued. Moreover, $G(x ; a, \underline{\theta})$ is a monotonically increasing function in $x$ with $G(a ; a, \underline{\theta})=0, G(\infty ; a, \underline{\theta})=\infty$ and $G^{\prime}(x ; a, \underline{\theta})$ denotes the derivative of $G(x ; a, \underline{\theta})$ with respect to $x$.

We note that (2.1) represents a family of lifetime distributions since it covers the following lifetime distributions as specific cases:

I. For $G(x ; a, \underline{\theta})=x$ and $a=0$, we get the one-parameter exponential distribution [Johnson and Kotz (1970, p.166)].

II. For $G(x ; a, \underline{\theta})=x^{p}(p>0)$ and $a=0$, it turns out to be Weibull distribution [Johnson and Kotz (1970, p.250)].

III. For $G(x ; a, \underline{\theta})=x^{2}$ and $a=0$, it gives Rayleigh distribution [Sinha (1986, p.200)].

IV. For $G(x ; a, \underline{\theta})=\log \left(1+x^{b}\right), b>0$ and $a=0$, it leads us to Burr distribution [Burr (1942) and Cislak and Burr (1968)].

V. For $(x ; a, \underline{\theta})=\log \left(\frac{x}{a}\right)$, we get Pareto distribution [Johnson and Kotz (1970, p.233)]. 
VI. For $G(x ; a, \underline{\theta})=\log \left(1+\frac{x}{v}\right), v>0$ and $a=0$, it is called Lomax (1954) distribution.

VII. For $G(x ; a, \underline{\theta})=\log \left(1+\frac{x^{b}}{v}\right), b>0, v>0$ and $a=0$, it becomes Burr distribution with scale parameter $v(>0)$ [Tadikamalla (1980)].

VIII. For $G(x ; a, \underline{\theta})=x^{\gamma} \exp (v x), \gamma>0, v>0$ and $a=0$, it gives the modified Weibull distribution of Lai et al (2003).

IX. For $G(x ; a, \underline{\theta})=(x-a)+\frac{v}{\lambda} \log \left(\frac{x+v}{a+\lambda}\right), v>0, \lambda>0$, we get the generalised Pareto distribution of Ljubo (1965).

X. For $G(x ; a, \underline{\theta})=b x+\frac{\theta}{2} x^{2}, \theta>0, b>0$ and $a=0$, we get the linear exponential distribution [Mahmoud and Al-Nagar (2009)].

XI. For $G(x ; a, \underline{\theta})=\left(1+x^{b}\right)^{\theta}-1, b>0, \theta>0$ and $a=0$, we get the generalised power Weibull distribution [Nikulin and Haghighi (2006)].

XII. For $G(x ; a, \underline{\theta})=\frac{\beta}{b}\left(e^{b x}-1\right), \beta>0, b>0$ and $a=0$, we get the Gompertz distribution [Khan and Zia (2009)].

XIII. For $G(x ; a, \underline{\theta})=\left(e^{x^{b}}-1\right), b>0$ and $a=0$, this gives Chen (2000) distribution.

XIV. For $G(x ; a, \underline{\theta})=(x-a)$, we get the two-parameter exponential distribution [Ahsanullah (1980)].

\section{Point Estimation Procedures}

Let $X_{1}, X_{2}, \ldots$ be an infinite sequence of independent and identically distributed (iid) rvs from (2.1). An observation $X_{j}$ will be called an upper record value (or simply a record) if its value exceeds that of all previous observations. Thus $X_{j}$ is a record if $X_{j}>X_{i}$ for every $i<j$.

The record time sequence $\left\{T_{n}, n \geq 0\right\}$ is defined as:

$\left\{\begin{array}{l}T_{0}=1 \quad ; \text { with probability } 1 \\ T_{n}=\min \left\{j: X_{j}>X_{T_{n-1}}\right\} ; n \geq 1\end{array}\right.$

The record value sequence $\left\{R_{n}\right\}$ is then defined by:

$R_{n}=X_{T_{n}} ; n=0,1,2, \ldots$

The likelihood function of the first $n+1$ upper record values $R_{0}, R_{1}, R_{2}, \ldots, R_{n}$ is:

$L\left(\lambda \mid R_{0}, R_{1}, R_{2}, \ldots, R_{n}\right)=f\left(R_{n} ; a, \lambda, \underline{\theta}\right) \prod_{i=0}^{n-1} \frac{f\left(R_{i} ; a, \lambda, \underline{\theta}\right)}{1-F\left(R_{i} ; a, \lambda, \underline{\theta}\right)}$

where $F(x ; a, \lambda, \underline{\theta})$ is the distribution function of $X$. It is easy to see that

$L\left(\lambda \mid R_{0}, R_{1}, R_{2}, \ldots, R_{n}\right)=\frac{\exp \left(\frac{-G\left(R_{n} ; a, \underline{\theta}\right)}{\lambda}\right)}{\lambda^{n+1}} \prod_{i=0}^{n} G^{\prime}\left(R_{i} ; a, \underline{\theta}\right)$.

The following theorem provides UMVUES of powers of $\lambda$. These estimators will be utilized to obtain the UMVUE of reliability functions.

Theorem 1: For $p \in(-\infty, \infty), p \neq 0$, the UMVUE of $\lambda^{-p}$ is given by: 
$\widetilde{\lambda-p}=\left\{\begin{array}{r}\left\{\frac{\Gamma(n+1)}{\Gamma(n-p+1)}\right\}\left(G\left(R_{n} ; a, \underline{\theta}\right)\right)^{-p} ; n>p-1 \\ 0 ; \text { otherwise }\end{array}\right.$

Proof: It follows from (3.1) and factorisation theorem [see Rohtagi and Saleh (2012, p.361)] that $G\left(R_{n} ; a, \underline{\theta}\right)$ is a sufficient statistic for $\lambda$ and the $p d f$ of $G\left(R_{n} ; a, \underline{\theta}\right)$ is:

$h\left(G\left(r_{n} ; a, \underline{\theta}\right) \mid \lambda\right)$

$$
=\frac{G\left(r_{n} ; a, \underline{\theta}\right)^{n}}{\Gamma(n+1) \lambda^{n+1}} \exp \left\{\frac{-G\left(r_{n} ; a, \underline{\theta}\right)}{\lambda}\right\}
$$

From (3.2), since the distribution of $R_{n}$ belongs to exponential family, it is also complete [see Rohtagi and Saleh (2012, p.367)]. The result now follows from (3.2) that $E\left[G\left(R_{n} ; a, \underline{\theta}\right)^{-p}\right]=\left\{\frac{\Gamma(n-p+1)}{\Gamma(n+1)}\right\} \lambda^{-p}$

In the following theorem, we obtain UMVUE of the reliability function.

Theorem 2: The UMVUE of the reliability function is

$\tilde{R}(t)=\left\{\begin{array}{cc}{\left[1-\frac{G(t ; a, \underline{\theta})}{G\left(R_{n} ; a, \underline{\theta}\right)}\right]^{n} ;} & G(t ; a, \underline{\theta})<G\left(R_{n} ; a, \underline{\theta}\right) \\ 0 ; & \text { otherwise }\end{array}\right.$

Proof: It is easy to see that

$$
\begin{aligned}
R(t) & =\exp \left\{\frac{-G(t ; a, \underline{\theta})}{\lambda}\right\} \\
& =\sum_{i=0}^{\infty} \frac{(-1)^{i}}{i !}\left\{\frac{G(t ; a, \underline{\theta})}{\lambda}\right\}^{i}
\end{aligned}
$$

Applying Theorem 1, it follows from (3.3) that

$$
\begin{aligned}
\tilde{R}(t) & =\sum_{i=0}^{\infty} \frac{(-1)^{i}}{i !}\{G(t ; a, \underline{\theta})\}^{i} \widetilde{\lambda^{-l}} \\
& =\sum_{i=0}^{n}(-1)^{i}\left(\begin{array}{l}
n \\
i
\end{array}\right)\left\{\frac{G(t ; a, \underline{\theta})}{G\left(R_{n} ; a, \underline{\theta}\right)}\right\}^{i}
\end{aligned}
$$

and the theorem follows.

The following corollary provides UMVUE of the sampled $p d f$. This estimator is derived with the help of Theorem 2 .

Corollary 1: The UMVUE of the sampled $p d f(2.1)$ at a specified point $x$ is $\tilde{f}(x ; a, \lambda, \underline{\theta})$

$=\left\{\begin{array}{cc}\frac{n G^{\prime}(x ; a, \underline{\theta})}{G\left(R_{n} ; a, \underline{\theta}\right)}\left[1-\frac{G(x ; a, \underline{\theta})}{G\left(R_{n} ; a, \underline{\theta}\right)}\right]^{\mathrm{n}-1} & ; G(x ; a, \underline{\theta})<G\left(R_{n} ; a, \underline{\theta}\right) \\ 0 & ; \text { otherwise }\end{array}\right.$

Proof: We note that the expectation of $\int_{t}^{\infty} f(x ; a, \lambda, \underline{\theta}) d x$ with respect to $R_{n}$ is $R(t)$. Hence, 
$\tilde{R}(t)=\int_{t}^{\infty} \tilde{f}(x ; a, \lambda, \underline{\theta}) d x$

The result follows from Theorem 2 .

In the following theorem, we obtain expression for the variance of $\tilde{R}(t)$, which will be needed to study its efficiency.

Theorem 3: The variance of $\tilde{R}(t)$ is given by:

$$
\begin{aligned}
\operatorname{Var}\{\tilde{R}(t)\}= & \frac{1}{n !}\left\{\frac{G(t ; a, \underline{\theta})}{\lambda}\right\}^{(n+1)} \exp \left\{\frac{-G(t ; a, \underline{\theta})}{\lambda}\right\}\left[\frac{\lambda a_{n}}{G(t ; a, \underline{\theta})}\right. \\
& -a_{n-1} \exp \left\{\frac{G(t ; a, \underline{\theta})}{\lambda}\right\} E_{i}\left(\frac{-G(t ; a, \underline{\theta})}{\lambda}\right) \\
& +\sum_{i=0}^{n-2} a_{i}\left\{\sum_{m=1}^{n-i-1} \frac{(m-1) !}{(n-i-1) !}\left(\frac{-G(t ; a, \underline{\theta})}{\lambda}\right)^{n-i-m-1}\right. \\
& \left.-\frac{1}{(n-i-1) !}\left(\frac{-G(t ; a, \underline{\theta})}{\lambda}\right)^{n-i-1} \exp \left(\frac{G(t ; a, \underline{\theta})}{\lambda}\right) E_{i}\left(\frac{-G(t ; a, \underline{\theta})}{\lambda}\right)\right\} \\
& \left.+\sum_{i=n+1}^{2 n} a_{i}(i-n) !\left(\frac{\lambda}{G(t ; a, \underline{\theta})}\right)^{i-n+1} \sum_{r=0}^{i-n} \frac{1}{r !}\left(\frac{G(t ; a, \underline{\theta})}{\lambda}\right)^{r}\right] \\
& -\exp \left\{\frac{-2 G(t ; a, \underline{\theta})}{\lambda}\right\},
\end{aligned}
$$

where $a_{i}=(-1)^{i}\left(\begin{array}{c}2 n \\ i\end{array}\right)$ and $-E_{i}(-x)=\int_{x}^{\infty} \frac{e^{-u}}{u} d u$.

Proof: Using (3.2) and Theorem 2,

$E\left\{\tilde{R}(t)^{2}\right\}$

$$
\begin{aligned}
& =\frac{1}{\Gamma(n+1) \lambda^{n+1}} \int_{G(t ; a, \underline{\theta})}^{\infty}\left[1-\frac{G(t ; a, \underline{\theta})}{G\left(r_{n} ; a, \underline{\theta}\right)}\right]^{2 n}\left\{G\left(r_{n} ; a, \underline{\theta}\right)\right\}^{n} \exp \left\{\frac{-G\left(r_{n} ; a, \underline{\theta}\right)}{\lambda}\right\} d G\left(r_{n} ; a, \underline{\theta}\right) \\
& =\frac{1}{\Gamma(n+1)}\left(\frac{G(t ; a, \underline{\theta})}{\lambda}\right)^{n+1} \exp \left(\frac{-G(t ; a, \underline{\theta})}{\lambda}\right) \int_{0}^{\infty} \frac{u^{2 n}}{(1+u)^{n}} \exp \left(\frac{-G(t ; a, \underline{\theta})}{\lambda} u\right) d u \\
& =\frac{1}{\Gamma(n+1)}\left(\frac{G(t ; a, \underline{\theta})}{\lambda}\right)^{n+1} \exp \left(\frac{-G(t ; a, \underline{\theta})}{\lambda}\right) I, \text { (say) }
\end{aligned}
$$

where

$$
\begin{aligned}
I & =\sum_{i=0}^{n} a_{i} \int_{0}^{\infty} \frac{1}{(u+1)^{n-i}} \exp \left(\frac{-G(t ; a, \underline{\theta})}{\lambda} u\right) d u \\
& +\sum_{i=n+1}^{2 n} a_{i} \int_{0}^{\infty}(u+1)^{i-n} \exp \left(\frac{-G(t ; a, \underline{\theta})}{\lambda} u\right) d u
\end{aligned}
$$


Using a result of Erdélyi (1954) that

$\int_{0}^{\infty} \frac{\exp (-u p)}{(u+a)^{n}} d u=\sum_{m=1}^{n-1} \frac{(m-1) !(-p)^{n-m-1}}{(n-1) ! a^{m}}-\frac{(-p)^{n-1}}{(n-1) !} \exp (a p) E_{i}(-a p)$

we have

$$
\begin{aligned}
& \int_{0}^{\infty} \frac{1}{(u+1)^{n-i}} \exp \left(\frac{-G(t ; a, \underline{\theta})}{\lambda} u\right) d u \\
& =\sum_{m=1}^{n-i-1} \frac{(m-1) !}{(n-1) !}\left(\frac{-G(t ; a, \underline{\theta})}{\lambda}\right)^{n-i-m-1} \\
& -\frac{1}{(n-i-1) !}\left(\frac{-G(t ; a, \underline{\theta})}{\lambda}\right)^{n-i-1} \exp \left(\frac{G(t ; a, \underline{\theta})}{\lambda}\right) E_{i}\left(\frac{-G(t ; a, \underline{\theta})}{\lambda}\right), i=0,1,2, \ldots n-2
\end{aligned}
$$

Furthermore,

$$
\begin{aligned}
& \int_{0}^{\infty} \frac{1}{(1+u)} \exp \left(\frac{-G(t ; a, \underline{\theta})}{\lambda} u\right) d u \\
& =\exp \left(\frac{G(t ; a, \underline{\theta})}{\lambda}\right) \int_{0}^{\infty} \frac{1}{1+u} \exp \left(\frac{-G(t ; a, \underline{\theta})}{\lambda}(1+u)\right) d u \\
& =\exp \left(\frac{G(t ; a, \underline{\theta})}{\lambda}\right) \int_{\left(\frac{G(t ; a, \underline{\theta})}{\lambda}\right)}^{\infty} \frac{e^{-z}}{z} d z \\
& =-\exp \left(\frac{G(t ; a, \underline{\theta})}{\lambda}\right) E_{i}\left(\frac{-G(t ; a, \underline{\theta})}{\lambda}\right) .
\end{aligned}
$$

We have

$$
\int_{0}^{\infty} \exp \left(\frac{-G(t ; a, \underline{\theta})}{\lambda} u\right) d u=\left(\frac{G(t ; a, \underline{\theta})}{\lambda}\right)
$$

Finally,

$$
\begin{aligned}
\int_{0}^{\infty}(1+u)^{i-n} \exp \left(\frac{-G(t ; a, \underline{\theta})}{\lambda} u\right) d u & =\sum_{r=0}^{i-n}\left(\begin{array}{c}
i-n \\
r
\end{array}\right) \int_{0}^{\infty} u^{i-n-r} \exp \left(\frac{-G(t ; a, \underline{\theta})}{\lambda} u\right) d u \\
& =\sum_{r=0}^{i-n}\left(\begin{array}{c}
i-n \\
r
\end{array}\right)\left\{\frac{\lambda}{G(t ; a, \underline{\theta})}\right\}^{i-n-r+1} \Gamma(i-n-r+1) .
\end{aligned}
$$

The theorem now follows on making substitutions from (3.7), (3.8), (3.9) and (3.10) in (3.6) and then using (3.5).

Let $X$ and $Y$ be two independent $r v s$ following the families of distributions $f_{1}\left(x ; a_{1}, \lambda_{1}, \underline{\theta_{1}}\right)$ and $f_{2}\left(y ; a_{2}, \lambda_{2}, \underline{\theta_{2}}\right)$ respectively. We consider the case when $X$ and $Y$ belong to different families of distributions, i.e. 


$$
f_{1}\left(x ; a_{1}, \lambda_{1}, \underline{\theta_{1}}\right)=\frac{G^{\prime}\left(x ; a_{1}, \underline{\theta_{1}}\right)}{\lambda_{1}} \exp \left\{\frac{-G\left(x ; a_{1}, \underline{\theta_{1}}\right)}{\lambda_{1}}\right\} ; \quad x>a_{1} \geq 0, \lambda_{1}>0
$$

and

$$
f_{2}\left(y ; a_{2}, \lambda_{2}, \underline{\theta_{2}}\right)=\frac{H^{\prime}\left(y ; a_{2}, \underline{\theta_{2}}\right)}{\lambda_{2}} \exp \left\{\frac{-H\left(y ; a_{2}, \underline{\theta_{2}}\right)}{\lambda_{2}}\right\} ; \quad y>a_{2} \geq 0, \lambda_{2}>0
$$

Let $\left\{R_{n}\right\}$ and $\left\{R_{m}^{*}\right\}$ be the record value sequences for $X^{\prime} s$ and $Y^{\prime} s$ respectively.

The following theorem provides the UMVUE of $P$ when $X$ and $Y$ belong to different families of distributions.

Theorem 4: The UMVUE of $P$ is given by

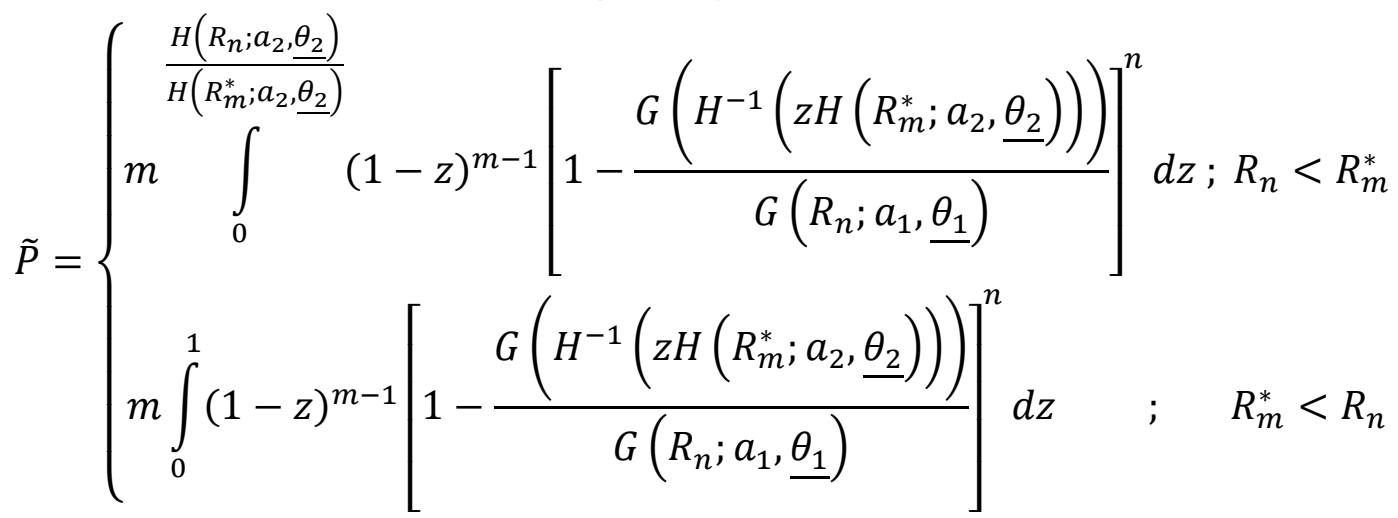

It follows from Corollary 1 that the UMVUES of $f_{1}\left(x ; a_{1}, \lambda_{1}, \underline{\theta_{1}}\right)$ and $f_{2}\left(y ; a_{2}, \lambda_{2}, \underline{\theta_{2}}\right)$ at specified points $x$ and $y$ are respectively:

$$
\begin{aligned}
& \widetilde{f}_{1}\left(x ; a_{1}, \lambda_{1}, \underline{\theta_{1}}\right) \\
& = \begin{cases}\frac{n G^{\prime}\left(x ; a_{1}, \underline{\theta_{1}}\right)}{G\left(R_{n} ; a_{1}, \underline{\theta_{1}}\right)}\left[1-\frac{G\left(x ; a_{1}, \underline{\theta_{1}}\right)}{G\left(R_{n} ; a_{1}, \underline{\theta_{1}}\right)}\right]_{0}^{\mathrm{n}-1} \quad ; G\left(x ; a_{1}, \underline{\theta_{1}}\right)<G\left(R_{n} ; a_{1}, \underline{\theta_{1}}\right) \\
\text {;otherwise }\end{cases}
\end{aligned}
$$

and

$$
\begin{aligned}
& \widetilde{f_{2}}\left(y ; a_{2}, \lambda_{2}, \underline{\theta_{2}}\right)
\end{aligned}
$$

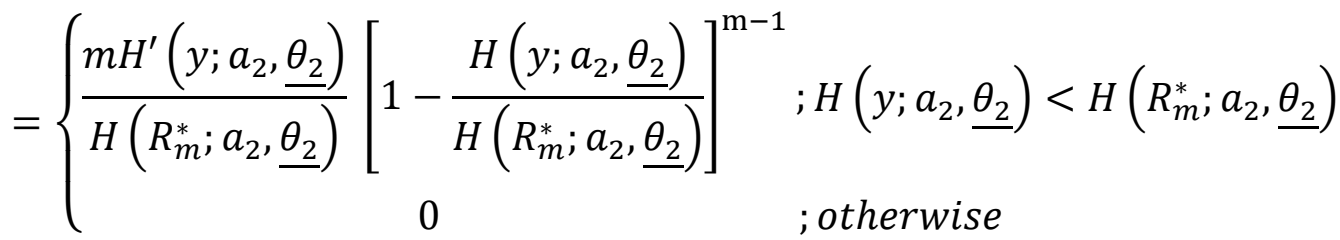

From the arguments similar to those used in the proof of Corollary 1,

$$
\tilde{P}=\int_{y=a_{2}}^{\infty} \int_{x=y}^{\infty} \widetilde{f}_{1}\left(x ; a_{1}, \lambda_{1}, \underline{\theta_{1}}\right) \widetilde{f}_{2}\left(y ; a_{2}, \lambda_{2}, \underline{\theta_{2}}\right) d x d y
$$




$$
\begin{aligned}
& =\int_{y=a_{2}}^{\infty} \tilde{R}_{1}(y)\left\{-\frac{d}{d y} \tilde{R}_{2}(y)\right\} d y \\
& =m \int_{y=a_{2}}^{\min \left[R_{n}, R_{m}^{*}\right]}\left[1-\frac{G\left(y ; a_{1}, \underline{\theta_{1}}\right)}{G\left(R_{n} ; a_{1}, \underline{\theta_{1}}\right)}\right]^{n}\left\{\frac{H^{\prime}\left(y ; a_{2}, \underline{\theta_{2}}\right)}{H\left(R_{m}^{*} ; a_{2}, \underline{\theta_{2}}\right)}\right\}\left[1-\frac{H\left(y ; a_{2}, \underline{\theta_{2}}\right)}{H\left(R_{m}^{*} ; a_{2}, \underline{\theta_{2}}\right)}\right]^{\mathrm{m}-1} d y
\end{aligned}
$$

The theorem now follows on considering the two cases and putting $H\left(R_{m}^{*} ; a_{2}, \underline{\theta_{2}}\right)^{-1} H\left(y ; a_{2}, \underline{\theta_{2}}\right)=z$

In the following theorem, we obtain the UMVUE of $P$ when $X$ and $Y$ belong to same families of distributions.

Theorem 5: When $X$ and $Y$ belong to same families of distributions,

$$
\tilde{P}= \begin{cases}\sum_{i=0}^{m-1} \frac{(-1)^{i} m ! n !}{(m-i-1) !(n+i+1) !}\left\{\frac{G\left(R_{n} ; a_{1}, \underline{\theta_{1}}\right)}{G\left(R_{m}^{*} ; a_{1}, \underline{\theta_{1}}\right)}\right\}^{i+1} & ; R_{n}<R_{m}^{*} \\ \sum_{i=0}^{n} \frac{(-1)^{i} n ! m !}{(n-i) !(m+i) !}\left\{\frac{\left.G\left(R_{m}^{*} ; a_{1}, \underline{\theta_{1}}\right)\right\}^{i}}{G\left(R_{n} ; a_{1}, \underline{\theta_{1}}\right)}\right. & ; R_{m}^{*}<R_{n}\end{cases}
$$

Proof: Taking $G(\cdot)=H(\cdot)$ in Theorem 4 , for $R_{n}<R_{m}^{*}$,

$$
\begin{aligned}
& \frac{G\left(R_{n} ; a_{1}, \underline{\theta_{1}}\right)}{G\left(R_{m}^{*} ; a_{1}, \underline{\theta_{1}}\right)} \\
& \tilde{P}=m \int_{0}^{G\left(R_{m}^{*} ; a_{1}, \underline{\theta_{1}}\right)}(1-z)^{m-1}\left\{1-\frac{z G\left(R_{m}^{*} ; a_{1}, \underline{\theta_{1}}\right)}{G\left(R_{n} ; a_{1}, \underline{\theta_{1}}\right)}\right\}^{n} d z \\
& =m\left\{\frac{G\left(R_{n} ; a_{1}, \underline{\theta_{1}}\right)}{G\left(R_{m}^{*} ; a_{1}, \underline{\theta_{1}}\right)}\right\} \int_{0}^{1}\left\{1-\frac{u G\left(R_{n} ; a_{1}, \underline{\theta_{1}}\right)}{G\left(R_{m}^{*} ; a_{1}, \underline{\theta_{1}}\right)}\right\}^{m-1}(1-u)^{n} d u \\
& =m \sum_{i=0}^{m-1}(-1)^{i}\left(\begin{array}{c}
m-1 \\
i
\end{array}\right)\left\{\frac{G\left(R_{n} ; a_{1}, \underline{\theta_{1}}\right)}{G\left(R_{m}^{*} ; a_{1}, \underline{\theta_{1}}\right)}\right\}^{i+1} \int_{0}^{1} u^{i}(1-u)^{n} d u
\end{aligned}
$$

and the first assertion follows. Similarly, we can prove the second assertion.

The following theorem provides the MLE of $R(t)$.

Theorem 6: The MLE of $R(t)$ is given by:

$\hat{R}(t)=\exp \left\{\frac{-(n+1) G(t ; a, \underline{\theta})}{G\left(R_{n} ; a, \underline{\theta}\right)}\right\}$

Proof: It can be easily seen from (3.1) that the MLE of $\lambda$ is $\hat{\lambda}=\frac{G\left(R_{n} ; a, \underline{\theta}\right)}{n+1}$. The theorem now follows from invariance property of MLE. 
In the following corollary, we obtain the MLE of sampled $p d f$ with the help of Theorem 6. This will be used to obtain MLE of $P$.

Corollary 2: The MLE of $f(x ; a, \lambda, \underline{\theta})$ at a specified point $x$ is

$\hat{f}(x ; a, \lambda, \underline{\theta})=\frac{(n+1) G^{\prime}(x ; a, \underline{\theta})}{G\left(R_{n} ; a, \underline{\theta}\right)} \exp \left\{\frac{-(n+1) G(x ; a, \underline{\theta})}{G\left(R_{n} ; a, \underline{\theta}\right)}\right\}$

Proof: The result follows from Theorem 6 on using the fact that $\hat{f}(x ; a, \lambda, \underline{\theta})=-\frac{d}{d t} \hat{R}(t)$.

In the following theorem, we obtain the expression for variance of $\hat{R}(t)$.

Theorem 7: The variance of $\hat{R}(t)$ is given by:

$$
\begin{aligned}
\operatorname{Var}\{\hat{R}(t)\}= & \frac{2}{n !}\left\{\frac{2(n+1) G(t ; a, \underline{\theta})}{\lambda}\right\}^{\frac{n+1}{2}} K_{n+1}\left(2 \sqrt{\frac{2(n+1) G(t ; a, \underline{\theta})}{\lambda}}\right) \\
& -\left[\frac{2}{n !}\left\{\frac{(n+1) G(t ; a, \underline{\theta})}{\lambda}\right\}^{\frac{n+1}{2}} K_{n+1}\left(2 \sqrt{\frac{(n+1) G(t ; a, \underline{\theta})}{\lambda}}\right)\right]^{2}
\end{aligned}
$$

where $K_{r}(\cdot)$ is modified Bessel function of second kind of order $r$.

Proof: Using (3.2) and Theorem 6, we have

$E\{\hat{R}(t)\}$

$$
\begin{aligned}
& =\frac{1}{\lambda^{n+1} \Gamma(n+1)} \int_{0}^{\infty} \exp \left[-\left\{\frac{G\left(R_{n} ; a, \underline{\theta}\right)}{\lambda}+\frac{(n+1) G(t ; a, \underline{\theta})}{G\left(R_{n} ; a, \underline{\theta}\right)}\right\}\right]\left\{G\left(R_{n} ; a, \underline{\theta}\right)\right\}^{n} d G\left(R_{n} ; a, \underline{\theta}\right) \\
& =\frac{1}{\Gamma(n+1)} \int_{0}^{\infty} \exp \left[-\left\{y+\frac{(n+1) G(t ; a, \underline{\theta})}{\lambda y}\right\}\right] y^{n} d y
\end{aligned}
$$

Applying a result of Watson (1952) that

$$
\int_{0}^{\infty} u^{-m} \exp \left\{-\left(a u+\frac{b}{u}\right)\right\} d u=2\left(\frac{a}{b}\right)^{\frac{m-1}{2}} K_{m-1}(2 \sqrt{a b})
$$

[it is to be noted that $K_{-m}(\cdot)=K_{m}(\cdot)$ for $m=0,1,2, \ldots$, we obtain from (3.11) that

$$
E\{\hat{R}(t)\}=\frac{2}{n !}\left\{\frac{(n+1) G(t ; a, \underline{\theta})}{\lambda}\right\}^{\frac{n+1}{2}} K_{n+1}\left(2 \sqrt{\frac{(n+1) G(t ; a, \underline{\theta})}{\lambda}}\right)
$$

Similarly, we can obtain the expression for $E\left\{\hat{R}(t)^{2}\right\}$ and the result follows.

The following theorem provides MLE of $P$ when $X$ and $Y$ belong to different families of distributions.

Theorem 8: The MLE of $P$ when $X$ and $Y$ belong to different families of distributions, is 


$$
\hat{P}=\int_{0}^{\infty} e^{-z} \exp \left\{\frac{-(n+1)}{G\left(R_{n} ; a_{1}, \underline{\theta_{1}}\right)} G\left(H^{-1}\left(\frac{z H\left(R_{m}^{*} ; a_{2}, \underline{\theta_{2}}\right.}{m+1}\right)\right)\right\} d z
$$

Proof: We have,

$$
\begin{aligned}
& \hat{P}=\int_{y=a_{2}}^{\infty} \int_{x=y}^{\infty} \widehat{f}_{1}\left(x ; a_{1}, \lambda_{1}, \underline{\theta_{1}}\right) \widehat{f}_{2}\left(y ; a_{2}, \lambda_{2}, \underline{\theta_{2}}\right) d x d y \\
& =\int_{y=a_{2}}^{\infty} \hat{R}_{1}\left(y ; a_{1}, \underline{\theta_{1}}\right)\left\{-\frac{d}{d y} \hat{R}_{2}\left(y ; a_{2}, \underline{\theta_{2}}\right)\right\} d y \\
& =\int_{y=a_{2}}^{\infty} \exp \left\{\frac{-(n+1) G\left(y ; a_{1}, \underline{\theta_{1}}\right)}{G\left(R_{n} ; a_{1}, \underline{\theta_{1}}\right)}\right\}\left\{\frac{(m+1) H^{\prime}\left(y ; a_{2}, \underline{\theta_{2}}\right)}{H\left(R_{m}^{*} ; a_{2}, \underline{\theta_{2}}\right)}\right\} \exp \left\{\frac{-(m+1) H\left(y ; a_{2}, \underline{\theta_{2}}\right)}{H\left(R_{m}^{*} ; a_{2}, \underline{\theta_{2}}\right)}\right\} d y
\end{aligned}
$$

The result now follows on putting $\left\{\frac{(m+1) H\left(y ; a_{2}, \underline{\theta_{2}}\right)}{H\left(R_{m}^{*} ; a_{2}, \underline{\theta_{2}}\right)}\right\}=z$.

The following theorem provides MLE of $P$ when $X$ and $Y$ belong to same families of distributions. The result follows from Theorem 8.

Theorem 9: When $X$ and $Y$ belong to same families of distributions, the MLE of $P$ is given by

$$
\hat{P}=\frac{(m+1) G\left(R_{n} ; a, \underline{\theta}\right)}{(m+1) G\left(R_{n} ; a, \underline{\theta}\right)+(n+1) G\left(R_{m}^{*} ; a, \underline{\theta}\right)}
$$

\section{Test Procedures For Various Hypotheses}

Suppose we have to test the hypothesis $H_{\mathrm{o}}: \lambda=\lambda_{\mathrm{o}}$ against $H_{1}: \lambda \neq \lambda_{\mathrm{o}}$. It follows from (3.1) that, under $H_{\mathrm{o}}$,

$$
\sup _{\Theta_{0}} L\left(\lambda \mid R_{0}, R_{1}, \ldots, R_{n}\right)=\frac{1}{\lambda_{\mathrm{o}}{ }^{n+1}} \exp \left\{\frac{-G\left(R_{n} ; a, \underline{\theta}\right)}{\lambda_{\mathrm{o}}}\right\} \prod_{i=0}^{n} G^{\prime}\left(R_{i} ; a, \underline{\theta}\right) ; \Theta_{\mathrm{o}}=\left\{\lambda: \lambda=\lambda_{\mathrm{o}}\right\}
$$

and

$$
\sup _{\Theta} L\left(\lambda \mid R_{0}, R_{1}, \ldots, R_{n}\right)=\left\{\frac{n+1}{G\left(R_{n} ; a, \underline{\theta}\right)}\right\}^{n+1} \exp (-(n+1)) \prod_{i=0}^{n} G^{\prime}\left(R_{i} ; a, \underline{\theta}\right) ; \Theta=\{\lambda: \lambda>0\}
$$

Therefore, the likelihood ratio (LR) is given by:

$$
\begin{aligned}
\varnothing\left(R_{0}, R_{1}, \ldots, R_{n}\right) & =\frac{\sup _{\Theta_{\mathrm{o}}} L\left(\lambda \mid R_{0}, R_{1}, \ldots, R_{n}\right)}{\sup _{\Theta} L\left(\lambda \mid R_{0}, R_{1}, \ldots, R_{n}\right)} \\
& =\left\{\frac{G\left(R_{n} ; a, \underline{\theta}\right)}{(n+1) \lambda_{\mathrm{o}}}\right\}^{n+1} \exp \left\{\frac{-G\left(R_{n} ; a, \underline{\theta}\right)}{\lambda_{\mathrm{o}}}+(n+1)\right\}
\end{aligned}
$$

We note that the first term on the right hand side of (4.1) is monotonically increasing and the second term is monotonically decreasing in $G\left(R_{n} ; a, \underline{\theta}\right)$. It follows from (3.2) that $2 \lambda_{\mathrm{o}}{ }^{-1} G\left(R_{n} ; a, \underline{\theta}\right) \sim \chi_{2(n+1)}^{2}$. Thus, the critical region is given by $\left\{0<G\left(R_{n} ; a, \underline{\theta}\right)<k_{\mathrm{o}}\right\} \cup$ 
$\left\{k_{\mathrm{o}}{ }^{\prime}<G\left(R_{n} ; a, \underline{\theta}\right)<\infty\right\}$, where $k_{\mathrm{o}}$ and $k_{\mathrm{o}}{ }^{\prime}$ are obtained such that $k_{\mathrm{o}}=\frac{\lambda_{\mathrm{o}}}{2} \chi_{2(n+1)}^{2}\left(\frac{\alpha}{2}\right)$ and $k_{\mathrm{o}}{ }^{\prime}=\frac{\lambda_{\mathrm{o}}}{2} \chi_{2(n+1)}^{2}\left(1-\frac{\alpha}{2}\right)$.

An important hypothesis in life-testing experiments is $H_{\mathrm{o}}: \lambda \geq \lambda_{\mathrm{o}}$ against $H_{1}: \lambda<\lambda_{\mathrm{o}}$. It follows from (3.1) that for $\lambda_{1}>\lambda_{2}$,

$\frac{L\left(\lambda_{1} \mid R_{0}, R_{1}, \ldots, R_{n}\right)}{L\left(\lambda_{2} \mid R_{0}, R_{1}, \ldots, R_{n}\right)}=\left(\frac{\lambda_{2}}{\lambda_{1}}\right)^{n+1} \exp \left\{\left(\frac{1}{\lambda_{2}}-\frac{1}{\lambda_{1}}\right) G\left(R_{n} ; a, \underline{\theta}\right)\right\}$

It follows from (4.2) that the family of distributions $f(x ; a, \lambda, \underline{\theta})$ has monotone likelihood ratio in $G\left(R_{n} ; a, \underline{\theta}\right)$. Thus, the uniformly most powerful critical region for testing $H_{\mathrm{o}}$ against $H_{1}$ is given by [see Lehmann $(1959$, p.88)]

$\emptyset\left(R_{0}, R_{1}, \ldots, R_{n}\right)=\left\{\begin{array}{rrr}1 & ; G\left(R_{n} ; a, \underline{\theta}\right) \leq k_{\mathrm{o}}^{\prime \prime} \\ 0 \quad ; & \text { otherwise }\end{array}\right.$

where $k_{\mathrm{o}}^{\prime \prime}=\frac{\lambda_{\mathrm{o}}}{2} \chi_{2(n+1)}^{2}(\alpha)$.

It can be seen that when $X$ and $Y$ belong to same families of distributions,

$P=\frac{\lambda_{1}}{\lambda_{1}+\lambda_{2}}$

Suppose we want to test $H_{\mathrm{o}}: P=P_{\mathrm{o}}$ against $H_{1}: P \neq P_{\mathrm{o}}$. It follows that $H_{\mathrm{o}}$ is equivalent to $\lambda_{1}=k \lambda_{2}$ where $k=\frac{P_{\mathrm{o}}}{1-P_{\mathrm{o}}}$. Thus, $H_{\mathrm{o}}: \lambda_{1}=k \lambda_{2}$ and $H_{1}: \lambda_{1} \neq k \lambda_{2}$.

It can be shown that, under $H_{\mathrm{o}}$,

$\hat{\lambda}_{1}=\frac{G\left(R_{n} ; a, \underline{\theta}\right)+k H\left(R_{m}^{*} ; a, \underline{\theta}\right)}{n+m+2}$

and

$\hat{\lambda}_{2}=\frac{G\left(R_{n} ; a, \underline{\theta}\right)+k H\left(R_{m}^{*} ; a, \underline{\theta}\right)}{k(n+m+2)}$

For a generic constant $K$,

$L\left(\lambda_{1}, \lambda_{2} \mid R_{0}, R_{1}, \ldots, R_{n}, R_{0}^{*}, R_{1}^{*}, \ldots, R_{m}^{*}\right)=\frac{K}{\lambda_{1}^{n+1} \lambda_{2}^{m+1}} \exp \left\{-\left(\frac{G\left(R_{n} ; a, \underline{\theta}\right)}{\lambda_{1}}+\frac{H\left(R_{m}^{*} ; a, \underline{\theta}\right)}{\lambda_{2}}\right)\right\}$

Thus,

$\sup _{\Theta_{\mathrm{o}}} L\left(\lambda_{1}, \lambda_{2} \mid R_{0}, R_{1}, \ldots, R_{n}, R_{0}^{*}, R_{1}^{*}, \ldots, R_{m}^{*}\right)$

$=\frac{K}{\left\{\frac{G\left(R_{n} ; a, \underline{\theta}\right)}{k}+H\left(R_{m}^{*} ; a, \underline{\theta}\right)\right\}^{n+m+2}} \exp \{-(n+m+2)\} ; \Theta_{0}=\left\{\lambda_{1}, \lambda_{2}: \lambda_{1}=k \lambda_{2}\right\}$

and

$\sup _{\Theta} L\left(\lambda_{1}, \lambda_{2} \mid R_{0}, R_{1}, \ldots, R_{n}, R_{0}^{*}, R_{1}^{*}, \ldots, R_{m}^{*}\right)$

$=\frac{K}{\left\{G\left(R_{n} ; a, \underline{\theta}\right)\right\}^{n+1}\left\{H\left(R_{m}^{*} ; a, \underline{\theta}\right)\right\}^{m+1}} \exp \{-(n+m+2)\} ; \Theta=\left\{\lambda_{1}, \lambda_{2}: \lambda_{1}>0, \lambda_{2}>0\right\}$

From (4.3) and (4.4), the LR is: 
$\varnothing\left(R_{0}, R_{1}, \ldots, R_{n}, R_{0}^{*}, R_{1}^{*}, \ldots, R_{m}^{*}\right)=\frac{K\left\{\frac{H\left(R_{m}^{*} ; a, \underline{\theta}\right)}{G\left(R_{n} ; a, \underline{\theta}\right)}\right\}^{m+1}}{\left\{1+\frac{k H\left(R_{m}^{*} ; a, \underline{\theta}\right)}{G\left(R_{n} ; a, \underline{\theta}\right)}\right\}^{n+m+2}}$

Denoting by $F_{a, b}(\cdot)$, the $F$ - Statistic with $(a, b)$ degrees of freedom and using the fact that $\frac{G\left(R_{n} ; a, \underline{\theta}\right)}{H\left(R_{m}^{*} ; a, \underline{\theta}\right)} \sim \frac{(n+1) \lambda_{1}}{(m+1) \lambda_{2}} F_{2(n+1), 2(m+1)}$, the critical region is given by $\left\{\frac{G\left(R_{n} ; a, \underline{\theta}\right)}{H\left(R_{m}^{*} ; a, \underline{\theta}\right)}<k_{2}\right\} U$ $\left\{\frac{G\left(R_{n} ; a, \underline{\theta}\right)}{H\left(R_{m}^{*} ; a, \underline{\theta}\right)}>k_{2}{ }^{\prime}\right\}$, where $k_{2}=\frac{k(n+1)}{(m+1)} F_{2(n+1), 2(m+1)}\left(\frac{\alpha}{2}\right)$ and $k_{2}{ }^{\prime}=\frac{k(n+1)}{(m+1)} F_{2(n+1), 2(m+1)}(1-$ $\left.\frac{\alpha}{2}\right)$.

\section{Numerical Findings}

\subsection{Real Data}

We consider the real data set which was also used in Lawless (1982, p. 185). These data are from Nelson (1982), concerning the data on time to breakdown of an insulating fluid between electrodes at a voltage of $34 \mathrm{kV}$ (minutes). The 19 times to breakdown are:

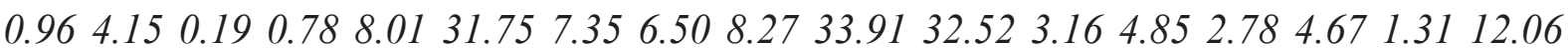
36.7172 .89

Therefore, we observe the following 7 upper record values:

\subsection{4 .158 .0131 .7533 .9136 .7172 .89}

We first apply Kolmogorov-Smirnov (K-S) test and Chi-Square test to check whether for a fixed voltage level, time to breakdown has a Weibull distribution. Considering Weibull distribution as a lifetime model for the complete data, the computed K-S statistic is 0.1616 with a $p$-value of 0.6462 . The computed Chi-Square statistic is 0.5369 with a $p$-value of0.4637. Both the tests indicate that Weibull Distribution is suitable for the data.

Using the method of Profile Log-likelihood before applying Newton-Raphson method, the Maximum Likelihood estimates of the parameters of Weibull distribution with scale parameter $\lambda$ and shape parameter $p$ obtained are $\hat{p}=0.7708, \hat{\lambda}=6.8865$. Based on these upper record values, $R_{n}=R_{6}=72.89, G\left(R_{n}\right)=G\left(R_{6}\right)=27.2762$, reliability function $R(t)_{t=2}=0.7894$, UMVUE of reliability function, $\tilde{R}(t)_{t=2}=0.7345, \quad$ and $\quad$ MLE $\quad$ of reliability function, $\hat{R}(t)_{t=2}=0.7041$.

Now we present a data analysis of the strength data reported by Badar and Priest(1982). This data represents the strength measured in GPA for single carbon fibers and impregnated 1000carbon fiber tows. Single fibers were tested under tension at gauge lengths of $20 \mathrm{~mm}$ (Data Set 1) and 10mm (Data Set 2) with sample sizes 69 and 63 respectively. These data have been used previously by Raqab and Kundu (2005), Kundu and Gupta (2006), Kundu and Raqab (2009) and Asgharzadeh et al (2011). Kundu and Gupta (2006) analyzed these data sets using two-parameter Weibull distribution after subtracting 0.75 from both these data sets. After subtracting 0.75 from all the points of these data sets, Kundu and Gupta (2006) observed that the Weibull distributions with equal shape parameters fit to both these data sets. 
Data Set 1 (gauge length of $20 \mathrm{~mm}$ ):

1.3121 .3141 .4791 .5521 .7001 .8031 .8611 .8651 .9441 .9581 .9661 .9972 .0062 .0212 .027

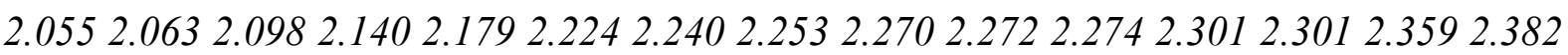

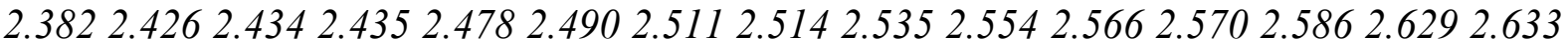
2.6422 .6482 .6842 .6972 .7262 .7702 .7732 .8002 .8092 .8182 .8212 .8482 .8802 .9543 .012 3.0673 .0843 .0903 .0963 .1283 .2333 .4333 .5853 .585

Data Set 2 (gauge length of $10 \mathrm{~mm}$ ):

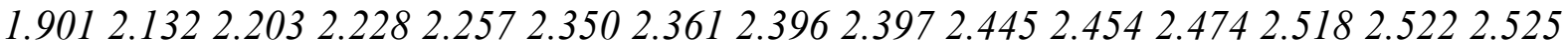

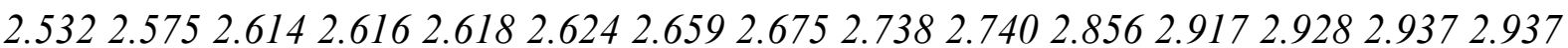
$2.9772 .9963 .0303 .1253 .1393 .1453 .2203 .2233 .2353 .243 \quad 3.2643 .2723 .2943 .3323 .346$ 3.3773 .4083 .4353 .4933 .5013 .5373 .5543 .5623 .6283 .8523 .8713 .8863 .9714 .0244 .027 4.2254 .3955 .020

Therefore, we observe the following upper record values:

Set of 66 record values from data set 1 :

$\begin{array}{lllllllllllll}1.3120 & 1.3140 & 1.4790 & 1.5520 & 1.7000 & 1.803 & 1.8610 & 1.8650 & 1.9440 & 1.9580 & 1.9660 & 1.9970\end{array}$ $\begin{array}{llllllllllll}2.0060 & 2.0210 & 2.0270 & 2.0550 & 2.0630 & 2.098 & 2.1400 & 2.1790 & 2.2240 & 2.2400 & 2.2530 & 2.2700\end{array}$

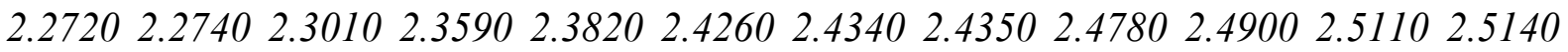
$\begin{array}{llllllllllll}2.5350 & 2.5540 & 2.5660 & 2.5700 & 2.5860 & 2.6290 & 2.6330 & 2.6420 & 2.6480 & 2.6840 & 2.6970 & 2.7260\end{array}$

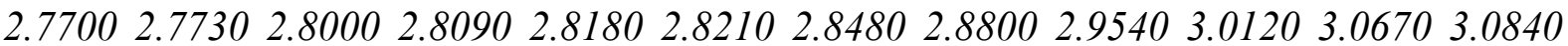
3.09003 .09603 .12803 .23303 .43303 .5850

Set of 62 record values from data set 2:

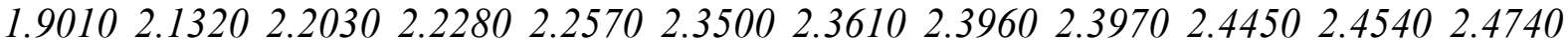

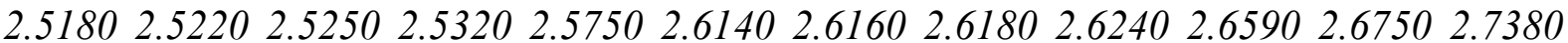

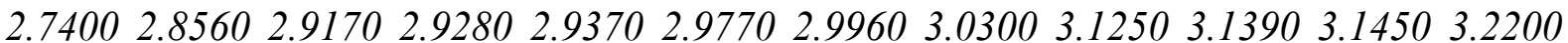

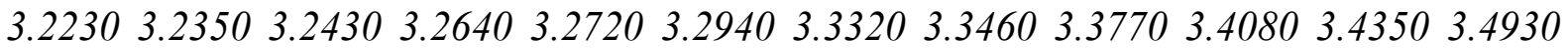

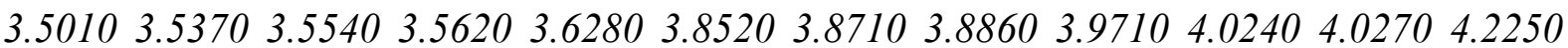
4.39505 .0200

Using the method of Profile Log-likelihood before applying Newton-Raphson method, the Maximum Likelihood estimates of the parameters of Weibull distribution fitting data set 1 with scale parameter $\lambda_{x}$ and shape parameter $p_{x}$ are $\widehat{\lambda_{x}}=214.1314$ and $\widehat{p_{x}}=$ 5.5049 respectively. Similarly, the Maximum Likelihood estimates of the parameters of Weibull distribution fitting data set 2 with scale parameter $\lambda_{y}$ and shape parameter $p_{y}$ are $\widehat{\lambda_{y}}=424.5736$ and $\widehat{p_{y}}=5.0494$ respectively. Based on the upper record values, $R_{n}=$ $R_{65}=3.5850, G\left(R_{n}\right)=G\left(R_{65}\right)=2.1872 e+03, R_{m}=R_{61}=5.02, \quad G\left(R_{m}\right)=G\left(R_{61}\right)=$ 9.4361e + 03.The UMVUE of stress-strength reliability, $\widetilde{P}=0.1772$ and MLE of stressstrength reliability, $\widehat{P}=0.1788$.

\subsection{Simulation Studies}


In order to obtain estimates under this scheme, we have generated (by inverse cumulative density method) $10,00,000$ samples of size 100 each from the distribution given in (2.1) with $(x ; a, \underline{\theta})=x^{p}, a=0, p=2, \lambda=5$. Assuming the data represents the life-span of items in hours, for $t=1$ and fixing the no. of record values to be $7(n=6)$, the no. of samples obtained are 1,18,282. $G\left(R_{n}\right)=25.9493, R(t)=0.8187$, MLE of $\lambda: \hat{\lambda}=$ 5.1899, UMVUE of $\lambda: \tilde{\lambda}=5.1899$, MLE of $R(t): \hat{R}(t)=0.8545$, UMVUE of $R(t): \tilde{R}(t)=$ 0.8247 , Variance of UMVUE of $R(t): \operatorname{Var}[\tilde{R}(t)]=0.003508$, MSE of MLE of $R(t): \operatorname{MSE}[\hat{R}(t)]=0.006613$.

In order to obtain the estimate of $P$ under this scheme, we have generated 10,000 samples of size 100 each from the distribution of $X$ and $Y$ when they belong to the same family of distributions. The samples are independently generated from (2.1) with $G(x ; a, \underline{\theta})=x^{p}$, $a=0, p=2, \lambda=5.5$. Fixing the no. of records from distribution of $X$ to be $n=5$ and the no. of records from distribution of $Y$ to be $m=7$. It can be easily shown that $P=$ $P(X>Y)=\frac{1}{2}$. The UMVUE of $P: \tilde{P}=0.5543$ and MLE of $P: \hat{P}=0.5447$. Now, when $X$ and $Y$ belong to different families of distributions, samples are independently generated from (2.1) with $G\left(x ; a_{1}, \underline{\theta_{1}}\right)=x^{p_{1}}, a_{1}=0, p_{1}=2, \lambda_{1}=5, H\left(y ; a_{2}, \underline{\theta_{2}}\right)=y^{p_{2}}, a_{2}=0, p_{2}=3$, $\lambda_{2}=7$. Fixing the no. of records from distribution of $X$ to be $n=10$ and the no. of records from distribution of $Y$ to be $m=12$. It can be easily shown that $P=$ $\frac{p_{2}}{\lambda_{2}} \int_{y=0}^{\infty} y^{p_{2}-1} \exp \left(-\frac{y^{p_{2}}}{\lambda_{2}}-\frac{y^{p_{1}}}{\lambda_{1}}\right) d y=0.5632$. The UMVUE of $P: \tilde{P}=$ 0.5301 and MLE of $P: \hat{P}=0.5209$.

In order to investigate the performance of the estimators obtained under this scheme, we have evaluated $\operatorname{Var}(\tilde{R}(t))$ and $\operatorname{MSE}(\hat{R}(t))$ for $G(x ; a, \underline{\theta})=x^{p}, a=0, p=0.77, \lambda=6.88$. Table 1 gives $\operatorname{Var}(\tilde{R}(t))$ and $\operatorname{MSE}(\hat{R}(t))$ for $t=1(1) 30$ and $n=8(1) 17$. Figure 1 compares the variance UMVUE of reliability function with the mean square error of MLE of reliability function calculated in Table 1 as time $t$ increases for $n=17$.

In the theory developed in Section 4, for testing the hypothesis $H_{\mathrm{o}}: \lambda=\lambda_{\mathrm{o}}$ against $H_{1}: \lambda \neq \lambda_{\mathrm{o}}$ under this scheme, we have considered the following sample.

Sample 1:

$\begin{array}{lllllllll}61.0260 & 67.1303 & 70.4844 & 81.8177 & 101.8750 & 105.5080 & 110.9864 & 123.1468 & 164.0256\end{array}$ $200.8713281 .5592 \quad 295.6992303 .7137318 .1099368 .2300$

Now with the help of Chi-Square tables at 5\% level of significance, we obtained $k_{\mathrm{o}}=$ 57.7602 and $k_{\mathrm{o}}{ }^{\prime}=161.6086$. Hence, in this case we may accept $H_{\mathrm{o}}$ at $5 \%$ level of significance since $G\left(R_{14}\right)=94.6045$.

Again, for testing $H_{\mathrm{o}}: \lambda \leq \lambda_{\mathrm{o}}$ against $H_{1}: \lambda>\lambda_{\mathrm{o}}$. we have considered the above Sample 1 . Now at $5 \%$ level of significance we obtained $k_{\mathrm{o}}{ }^{\prime \prime}=63.6147$ and hence, in this case we may accept $H_{\mathrm{o}}$ at $5 \%$ level of significance since $G\left(R_{14}\right)=94.6045$. 
In order to test $H_{\mathrm{o}}: P=P_{\mathrm{o}}$ against $H_{1}: P \neq P_{\mathrm{o}}$.under this scheme, we have considered the following Sample $X$ and Sample $Y$.

Sample $X$ :

$\begin{array}{llllllllll}1.3557 & 2.0975 & 2.1051 & 2.1916 & 2.3850 & 2.4133 & 2.4296 & 2.5964 & 2.7435 & 2.8080\end{array}$

$2.8404 \quad 2.87192 .9337 \quad 3.0365$

Sample $Y$ :

$\begin{array}{llllllllll}0.9105 & 1.4416 & 1.5719 & 1.8083 & 1.8614 & 1.8779 & 1.8879 & 1.8998 & 1.9696 & 2.1518\end{array}$

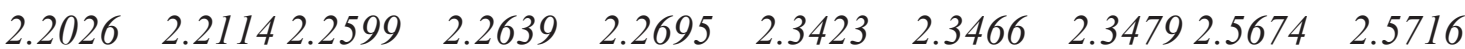

For these two samples we obtained $\frac{G\left(R_{n}\right)}{G\left(R_{m}^{*}\right)}=0.7559$. Now, with the help of $F-$ tables at $5 \%$ level of significance, we obtained $k_{2}=0.2506$ and $k_{2}^{\prime}=1.0069$. Hence, in this case we may accept $H_{\mathrm{o}}$ at $5 \%$ level of significance. 
Table 1: Mean Square Error of MLE and UMVUE of Reliability function

\begin{tabular}{|c|c|c|c|c|c|c|c|c|c|c|}
\hline$n$ & \multicolumn{2}{|c|}{8} & \multicolumn{2}{|c|}{9} & \multicolumn{2}{|c|}{10} & \multicolumn{2}{|c|}{11} & \multicolumn{2}{|c|}{12} \\
\hline$t$ & $\operatorname{Var}[U M V U E(R(t))]$ & $\operatorname{MSE}[\operatorname{MLE}(R(t))]$ & $\operatorname{Var}[\operatorname{UMVUE}(\mathrm{R}(\mathrm{t}))]$ & $\operatorname{MSE}[\operatorname{MLE}(R(t))]$ & $\operatorname{Var}[\operatorname{UMVUE}(\mathrm{R}(\mathrm{t}))]$ & $\operatorname{MSE}[\operatorname{MLE}(R(t))]$ & $\operatorname{Var}[\operatorname{UMVUE}(R(t))]$ & $\operatorname{MSE}[\operatorname{MLE}(R(t))]$ & $\operatorname{Var}[\operatorname{UMVUE}(\mathrm{R}(\mathrm{t}))]$ & $\operatorname{MSE}[\operatorname{MLE}(R(t))]$ \\
\hline 1 & 0.00216 & 0.00642 & 0.00190 & 0.00684 & 0.00170 & 0.00272 & 0.00153 & 0.00336 & 0.00140 & 0.00203 \\
\hline 2 & 0.00497 & 0.01471 & 0.00439 & 0.01595 & 0.00393 & 0.00606 & 0.00356 & 0.00767 & 0.00325 & 0.00457 \\
\hline 3 & 0.00758 & 0.02236 & 0.00671 & 0.02460 & 0.00602 & 0.00900 & 0.00546 & 0.01159 & 0.00499 & 0.00681 \\
\hline 4 & 0.00979 & 0.02891 & 0.00869 & 0.03222 & 0.00781 & 0.01136 & 0.00710 & 0.01488 & 0.00650 & 0.00864 \\
\hline 5 & 0.01160 & 0.03430 & 0.01032 & 0.03868 & 0.00929 & 0.01319 & 0.00844 & 0.01752 & 0.00774 & 0.01006 \\
\hline 6 & 0.01302 & 0.03861 & 0.01160 & 0.04403 & 0.01045 & 0.01453 & 0.00951 & 0.01957 & 0.00873 & 0.01112 \\
\hline 7 & 0.01410 & 0.04197 & 0.01257 & 0.04834 & 0.01134 & 0.01546 & 0.01033 & 0.02109 & 0.00948 & 0.01186 \\
\hline 8 & 0.01488 & 0.04450 & 0.01328 & 0.05175 & 0.01200 & 0.01606 & 0.01093 & 0.02217 & 0.01004 & 0.01235 \\
\hline 9 & 0.01541 & 0.04633 & 0.01377 & 0.05436 & 0.01244 & 0.01638 & 0.01135 & 0.02287 & 0.01043 & 0.01262 \\
\hline 10 & 0.01572 & 0.04755 & 0.01406 & 0.05629 & 0.01271 & 0.01648 & 0.01160 & 0.02325 & 0.01067 & 0.01272 \\
\hline 11 & 0.01585 & 0.04827 & 0.01419 & 0.05761 & 0.01284 & 0.01641 & 0.01172 & 0.02339 & 0.01079 & 0.01268 \\
\hline 12 & 0.01584 & 0.04856 & 0.01419 & 0.05844 & 0.01285 & 0.01619 & 0.01174 & 0.02331 & 0.01080 & 0.01253 \\
\hline 13 & 0.01571 & 0.04851 & 0.01408 & 0.05883 & 0.01275 & 0.01588 & 0.01166 & 0.02306 & 0.01073 & 0.01230 \\
\hline 14 & 0.01548 & 0.04817 & 0.01388 & 0.05886 & 0.01258 & 0.01548 & 0.01150 & 0.02268 & 0.01059 & 0.01201 \\
\hline 15 & 0.01518 & 0.04759 & 0.01361 & 0.05858 & 0.01234 & 0.01502 & 0.01129 & 0.02219 & 0.01040 & 0.01166 \\
\hline 16 & 0.01481 & 0.04683 & 0.01329 & 0.05805 & 0.01205 & 0.01452 & 0.01103 & 0.02162 & 0.01016 & 0.01128 \\
\hline 17 & 0.01440 & 0.04591 & 0.01293 & 0.05731 & 0.01173 & 0.01399 & 0.01073 & 0.02099 & 0.00989 & 0.01088 \\
\hline 18 & 0.01395 & 0.04487 & 0.01253 & 0.05640 & 0.01137 & 0.01344 & 0.01040 & 0.02032 & 0.00959 & 0.01046 \\
\hline 19 & 0.01348 & 0.04374 & 0.01211 & 0.05534 & 0.01099 & 0.01288 & 0.01006 & 0.01961 & 0.00928 & 0.01003 \\
\hline 20 & 0.01300 & 0.04254 & 0.01167 & 0.05417 & 0.01060 & 0.01232 & 0.00970 & 0.01889 & 0.00895 & 0.00960 \\
\hline 21 & 0.01250 & 0.04128 & 0.01123 & 0.05291 & 0.01020 & 0.01176 & 0.00934 & 0.01815 & 0.00861 & 0.00917 \\
\hline 22 & 0.01200 & 0.04000 & 0.01078 & 0.05159 & 0.00979 & 0.01121 & 0.00897 & 0.01742 & 0.00827 & 0.00875 \\
\hline 23 & 0.01150 & 0.03869 & 0.01033 & 0.05021 & 0.00938 & 0.01068 & 0.00859 & 0.01668 & 0.00793 & 0.00833 \\
\hline 24 & 0.01100 & 0.03737 & 0.00989 & 0.04880 & 0.00898 & 0.01015 & 0.00823 & 0.01596 & 0.00759 & 0.00793 \\
\hline 25 & 0.01052 & 0.03606 & 0.00945 & 0.04737 & 0.00858 & 0.00965 & 0.00786 & 0.01525 & 0.00725 & 0.00754 \\
\hline 26 & 0.01004 & 0.03475 & 0.00902 & 0.04592 & 0.00819 & 0.00916 & 0.00750 & 0.01455 & 0.00692 & 0.00716 \\
\hline 27 & 0.00957 & 0.03346 & 0.00860 & 0.04447 & 0.00781 & 0.00869 & 0.00716 & 0.01387 & 0.00660 & 0.00679 \\
\hline 28 & 0.00912 & 0.03219 & 0.00820 & 0.04302 & 0.00744 & 0.00824 & 0.00682 & 0.01322 & 0.00629 & 0.00644 \\
\hline 29 & 0.00868 & 0.03094 & 0.00780 & 0.04159 & 0.00708 & 0.00781 & 0.00649 & 0.01258 & 0.00598 & 0.00611 \\
\hline 30 & 0.00825 & 0.02972 & 0.00742 & 0.04017 & 0.00674 & 0.00739 & 0.00617 & 0.01197 & 0.00569 & 0.00579 \\
\hline
\end{tabular}




\begin{tabular}{|c|c|c|c|c|c|c|c|c|c|c|}
\hline$n$ & \multicolumn{2}{|c|}{13} & \multicolumn{2}{|c|}{14} & \multicolumn{2}{|c|}{15} & \multicolumn{2}{|c|}{16} & \multicolumn{2}{|c|}{17} \\
\hline$t$ & $\operatorname{Var}[\operatorname{UMVUE}(R(t))]$ & $\operatorname{MSE}[\operatorname{MLE}(R(t))]$ & $\operatorname{Var}[\operatorname{UMVUE}(R(t))]$ & $\operatorname{MSE}[\operatorname{MLE}(R(t))]$ & $\operatorname{Var}[\operatorname{UMVUE}(\mathrm{R}(\mathrm{t}))]$ & $\operatorname{MSE}[\operatorname{MLE}(R(t))]$ & $\operatorname{Var}[\operatorname{UMVUE}(R(t))]$ & $\operatorname{MSE}[\operatorname{MLE}(R(t))]$ & $\operatorname{Var}[\operatorname{UMVUE}(R(t))]$ & $\operatorname{MSE}[\operatorname{MLE}(R(t))]$ \\
\hline 1 & 0.00128 & 0.00146 & 0.00119 & 0.00143 & 0.00110 & 0.00129 & 0.00103 & 0.00130 & 0.00097 & 0.00214 \\
\hline 2 & 0.00299 & 0.00328 & 0.00277 & 0.00327 & 0.00258 & 0.00297 & 0.00242 & 0.00300 & 0.00227 & 0.00496 \\
\hline 3 & 0.00460 & 0.00490 & 0.00427 & 0.00497 & 0.00398 & 0.00451 & 0.00372 & 0.00458 & 0.00350 & 0.00758 \\
\hline 4 & 0.00599 & 0.00623 & 0.00556 & 0.00640 & 0.00518 & 0.00582 & 0.00486 & 0.00593 & 0.00457 & 0.00981 \\
\hline 5 & 0.00714 & 0.00727 & 0.00663 & 0.00757 & 0.00619 & 0.00689 & 0.00580 & 0.00704 & 0.00546 & 0.01163 \\
\hline 6 & 0.00806 & 0.00805 & 0.00749 & 0.00849 & 0.00699 & 0.00772 & 0.00656 & 0.00792 & 0.00617 & 0.01306 \\
\hline 7 & 0.00877 & 0.00861 & 0.00815 & 0.00919 & 0.00761 & 0.00836 & 0.00714 & 0.00859 & 0.00672 & 0.01414 \\
\hline 8 & 0.00929 & 0.00899 & 0.00864 & 0.00969 & 0.00807 & 0.00883 & 0.00758 & 0.00908 & 0.00714 & 0.01491 \\
\hline 9 & 0.00965 & 0.00922 & 0.00898 & 0.01004 & 0.00839 & 0.00914 & 0.00788 & 0.00943 & 0.00743 & 0.01542 \\
\hline 10 & 0.00988 & 0.00933 & 0.00919 & 0.01025 & 0.00860 & 0.00934 & 0.00807 & 0.00964 & 0.00761 & 0.01572 \\
\hline 11 & 0.00999 & 0.00934 & 0.00930 & 0.01035 & 0.00870 & 0.00943 & 0.00817 & 0.00975 & 0.00770 & 0.01583 \\
\hline 12 & 0.01000 & 0.00926 & 0.00932 & 0.01035 & 0.00872 & 0.00943 & 0.00819 & 0.00976 & 0.00772 & 0.01580 \\
\hline 13 & 0.00994 & 0.00913 & 0.00926 & 0.01028 & 0.00867 & 0.00937 & 0.00815 & 0.00971 & 0.00768 & 0.01564 \\
\hline 14 & 0.00982 & 0.00895 & 0.00915 & 0.01015 & 0.00856 & 0.00925 & 0.00805 & 0.00959 & 0.00759 & 0.01539 \\
\hline 15 & 0.00964 & 0.00873 & 0.00898 & 0.00997 & 0.00841 & 0.00909 & 0.00791 & 0.00943 & 0.00746 & 0.01506 \\
\hline 16 & 0.00942 & 0.00848 & 0.00878 & 0.00976 & 0.00822 & 0.00889 & 0.00773 & 0.00923 & 0.00730 & 0.01467 \\
\hline 17 & 0.00917 & 0.00821 & 0.00855 & 0.00951 & 0.00801 & 0.00866 & 0.00753 & 0.00900 & 0.00711 & 0.01423 \\
\hline 18 & 0.00890 & 0.00793 & 0.00830 & 0.00924 & 0.00777 & 0.00842 & 0.00731 & 0.00874 & 0.00690 & 0.01377 \\
\hline 19 & 0.00861 & 0.00765 & 0.00802 & 0.00895 & 0.00752 & 0.00815 & 0.00707 & 0.00847 & 0.00667 & 0.01327 \\
\hline 20 & 0.00830 & 0.00735 & 0.00774 & 0.00865 & 0.00725 & 0.00788 & 0.00682 & 0.00819 & 0.00644 & 0.01277 \\
\hline 21 & 0.00799 & 0.00706 & 0.00745 & 0.00834 & 0.00698 & 0.00760 & 0.00657 & 0.00790 & 0.00620 & 0.01225 \\
\hline 22 & 0.00767 & 0.00677 & 0.00716 & 0.00804 & 0.00671 & 0.00732 & 0.00631 & 0.00761 & 0.00596 & 0.01174 \\
\hline 23 & 0.00736 & 0.00648 & 0.00686 & 0.00773 & 0.00643 & 0.00704 & 0.00605 & 0.00731 & 0.00571 & 0.01122 \\
\hline 24 & 0.00704 & 0.00620 & 0.00657 & 0.00742 & 0.00616 & 0.00676 & 0.00579 & 0.00702 & 0.00547 & 0.01071 \\
\hline 25 & 0.00673 & 0.00592 & 0.00628 & 0.00711 & 0.00589 & 0.00648 & 0.00554 & 0.00673 & 0.00523 & 0.01022 \\
\hline 26 & 0.00643 & 0.00565 & 0.00599 & 0.00681 & 0.00562 & 0.00621 & 0.00529 & 0.00644 & 0.00499 & 0.00973 \\
\hline 27 & 0.00613 & 0.00539 & 0.00572 & 0.00652 & 0.00536 & 0.00594 & 0.00504 & 0.00616 & 0.00476 & 0.00926 \\
\hline 28 & 0.00584 & 0.00514 & 0.00544 & 0.00623 & 0.00510 & 0.00568 & 0.00480 & 0.00589 & 0.00453 & 0.00880 \\
\hline 29 & 0.00555 & 0.00490 & 0.00518 & 0.00595 & 0.00486 & 0.00542 & 0.00457 & 0.00562 & 0.00431 & 0.00835 \\
\hline 30 & 0.00528 & 0.00466 & 0.00493 & 0.00568 & 0.00462 & 0.00518 & 0.00434 & 0.00536 & 0.00410 & 0.00793 \\
\hline
\end{tabular}




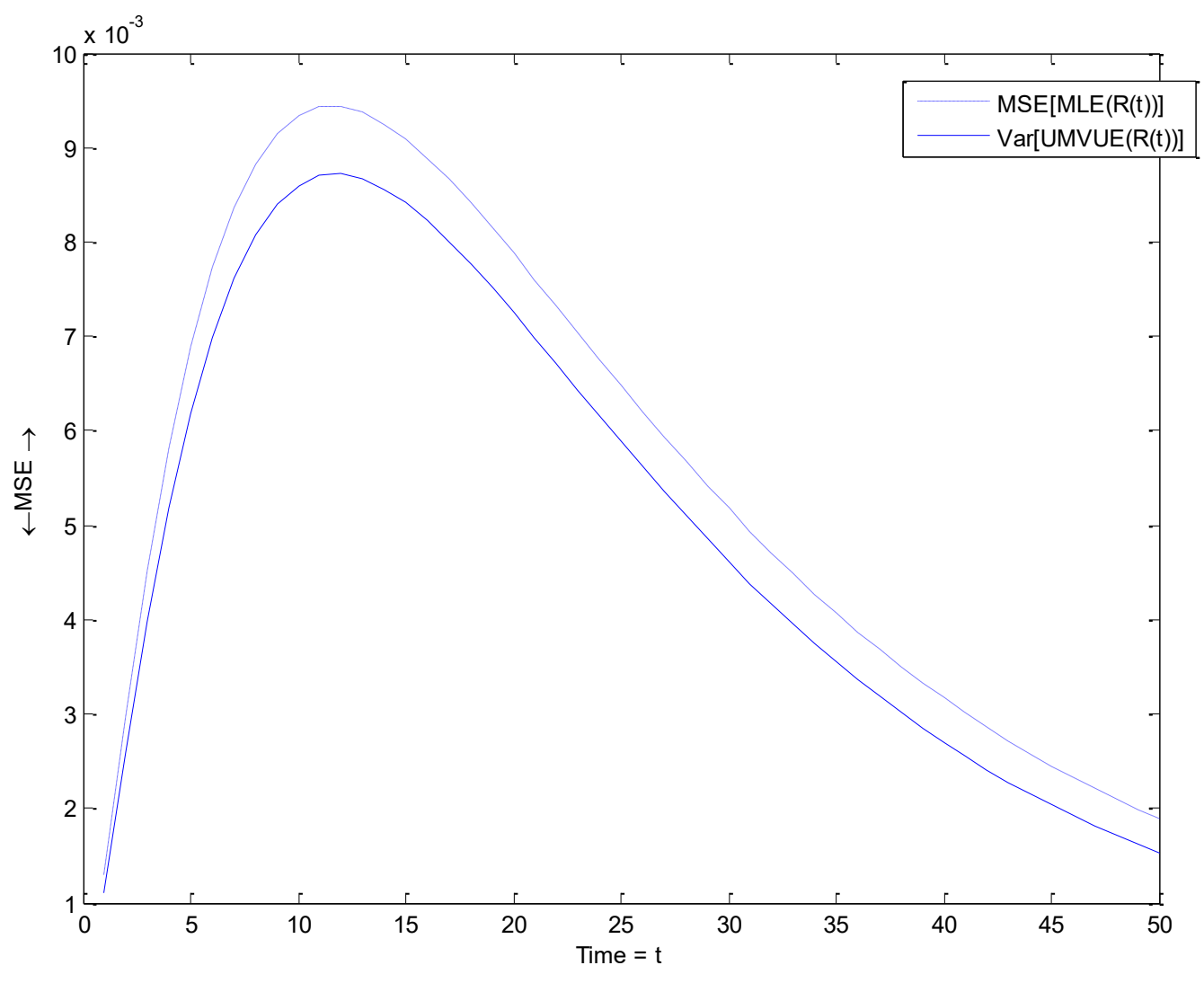

Figure 1: Mean Square Error of MLE and UMVUE of Reliability function for sample size

$$
n=17 \text {. }
$$

\section{Discussion}

A lot of work has been done in the literature to estimate and test the hypotheses for the reliability functions. In the present paper, we have proposed a family of lifetime distributions which covers as many as fourteen distributions as specific cases, which are useful in reliability theory. Based on record values, estimation and testing procedures are developed for this family of lifetime distributions. Thus, a unified theory is developed.

From Table 1, it is clear that at any given time $t$ and for any sample size $n$, the variance UMVUE of reliability function is always less than the mean square error of MLE of reliability function.

\section{Conclusion}

In Table 1, a comparative study of efficiencies of UMVUE and MLE of reliability function based on record values has been performed. It is clear from simulation results that UMVUES of the reliability function are more efficient than MLE of reliability function. Thus, a comparison between efficiencies of UMVUES and MLES has been discussed by estimating the sampled pdf to obtain the variance and mean square error of estimators and an interrelationship between efficiencies of the two estimators has been established by performing simulation studies. 


\section{Acknowledgement}

We are thankful to two reviewers for providing fruitful comments, which led to considerable improvement in the original paper.

\section{References}

1. Ahsanullah, M. (1980): Linear prediction of record values for the two parameter exponential distribution.Ann. Inst. Statist. Math., 32, Part A, 363-368.

2. Arnold, B. C., Balakrishan, N., Nagaraja, H. N. (1992): A First Course in Order Statistics. John Wiley \& Sons, New York.

3. Arashi,M., Emadi, M. (2008): Evidential inference based on record data and inter-record times. Stat.Papers, 13(8), 380-210.

4. Asgharzadeh, A., Valiollahi, R., Raqab. M. Z. (2011): Stress-strength reliability of Weibull distribution based on progressively censored samples. SORT, 35, 103-124.

5. Awad, A. M., Gharraf, M. K. (1986): Estimation of $\mathrm{P}(\mathrm{Y}<\mathrm{X})$ in the Burr case: A Comparative Study. Commun. Statist. - Simul., 15(2), 389-403.

6. Badar, M. G., Priest, A. M. (1982): Statistical aspects of fiber and bundle strength in hybrid composites. In: Hayashi, T., Kawata, K., Umekawa, S. (Eds.). Progress in Science and Engineering Composites, ICCM-IV, Tokyo, 1129-1136.

7. Balakrishan, N., Ahsanullah, M.,Chan, P.S. (1995): On the logistic record values and associated inference. Journal of Applied Statistical Science, 2, 233-248.

8. Bartholomew, D. J. (1957): A problem in life testing. Jour. Amer. Statist. Assoc., 52, 350355.

9. Bartholomew, D. J. (1963): The sampling distribution of an estimate arising in life testing. Technometrics, 5, 361-374.

10. Basu, A. P. (1964): Estimates of reliability for some distributions useful in life testing. Technometrics, 6, 215-219.

11. Belaghi, R. A., Arashi, M., Tabatabaey, S. M. M. (2015): On the Construction of Preliminary Test Estimator Based on Record Values for the Burr XII Model. Communications in Statistics - Theory and Methods, 44(1), 1-23, DOI:10.1080/03610926.2012.733473

12. Burr, I. W (1942): Cumulative Frequency Functions, The Annals of Mathematical Statistics $13(2), 215-232$

13. Burr, I.W., Cislak, P.J. (1968): On a general system of distributions: I. Its curve-shaped characteristics; II. The sample median, Journal of the American Statistical Association, 63, 627-635.

14. Chandler, K. N. (1952): The distribution and frequency of record values. Journal of the Royal Statistical Society, Series B, 14, 220-228.

15. Chao, A. (1982): On comparing estimators of $\operatorname{Pr}\{\mathrm{X}>\mathrm{Y}\}$ in the exponential case. IEEE Trans. Reliability, R-26, 389-392.

16. Chaturvedi,A and Kumari,T (2015): Estimation and testing procedures for the reliability functions of a family of lifetime distributions. interstat.statjournals.net/ YEAR/ 2015/ abstracts/ 1306001.php

17. Chaturvedi, A. and Pathak, A. (2012): Estimation of the reliability functions for exponentiated Weibull distribution. J. Stat. \& Appl, 7, 1-8.

18. Chaturvedi, A. and Pathak, A. (2013): Bayesian estimation procedures for three parameter exponentiated Weibull distribution under entropy loss function and type II censoring.interstat.statjournals.net/YEAR/2013/abstracts/1306001.php 
19. Chaturvedi, A. and Pathak, A. (2014): Estimation of the Reliability Function for fourParameter Exponentiated Generalized Lomax Distribution. IJSER, 5(1), 1171-1180.

20. Chaturvedi, A. and Rani, U. (1997): Estimation procedures for a family of density functions representing various life-testing models. Metrika, 46, 213-219.

21. Chaturvedi, A. and Rani, U. (1998): Classical and Bayesian reliability estimation of the generalized Maxwell failure distribution. Jour. Statist. Res., 32, 113-120.

22. Chaturvedi, A. and Singh, K. G. (2006): Bayesian estimation procedures for a family of lifetime distributions under squared-error and entropy losses. Metron, 64(2), 179-198.

23. Chaturvedi, A. and Singh, K. G. (2008): A family of lifetime distributions and related estimation and testing procedures for the reliability function. Jour. Appl. Statist. Sci., 16(2), 35-50.

24. Chaturvedi, A. and Surinder, K. (1999): Further remarks on estimating the reliability function of exponential distribution under type I and type II censorings. Brazilian Jour. Prob. Statist., 13, 29-39.

25. Chaturvedi., A. and Tomer, S. K. (2002): Classical and Bayesian reliability estimation of the negative binomial distribution. Jour. Applied Statist Sci., 11, 33-43.

26. Chaturvedi, A. and Tomer, S. K. (2003): UMVU estimation of the reliability function of the generalized life distributions. Statist. Papers, 44(3), 301-313.

27. Chen, Z. (2000): A new two- parameter lifetime distribution with bathtub shape or increasing failure rate function. Statistics \& Probability Letters, 49, 155-161.

28. Constantine, K., Karson, M. and Tse, S. K. (1986): Estimation of $\mathrm{P}(\mathrm{Y}<\mathrm{X})$ in the gamma case. Commun. Statist. - Simul., 15(2), 365-388.

29. Glick, N. (1978): Breaking records and breaking boards. American Mathematical Monthly, 85, 543-551.

30. Habibi Rad, A., Arghami, N. R., Ahmadi, J. (2006): Statistical evidence in experiments and in record values. Commun. Stat. Theo. Meth. 35(11), 1971-1983.

31. Johnson, N. L. (1975): Letter to the editor. Technometrics, 17, 393.

32. Johnson, N.L and Kotz, S. (1970): Distributions in Statistics-Continuous Univariate Distributons, Vol. 1, New York: John Wiley \& Sons, Vol. 1- 2.

33. Khan,R.U., Zia, B. (2009): Recurrence Relations for single and Product moments of record values from Gompertz distribution and a characterization. World Applied Sciences Journal, 7(10), 1331-1334.

34. Kelly, G. D., Kelly, J.A. Schucany, W. R. (1976): Efficient estimation of $\mathrm{P}(\mathrm{Y}<\mathrm{X})$ in the exponential case. Technometrics, 18, 359-360.

35. Kundu, D., Gupta, R. D. (2006): Estimation of $P(Y<X)$ for Weibull distributions. IEEE Transcations on Reliability, 55(2), 270-280.

36. Kundu, D., Raqab, M. Z. (2009): Estimation of $R=P(Y<X)$ for three parameter Weibull distribution. Statistics and Probability Letters, 79, 1839-1846.

37. Lai, C. D., Xie, M., Murthy, D. N. P. (2003): Modified Weibull model. IEEE Trans. Reliability, 52, 33-37.

38. Lawless, J. F. (1982): Statistical models and methods for lifetime data, John Wiley \& Sons, New York, 10(4), 316-317

39. Lehmann, E. L. (1959): Testing Statistical Hypotheses. John Wiley and Sons, New York.

40. Ljubo, M. (1965): Curves and concentration indices for certain generalized Pareto distributions. Statist. Rev., 15, 257-260.

41. Lomax, K. S. (1954): Business failures. Another example of the analysis of failure data. Jour. Amer. Statist. Assoc., 49, 847-852.

42. Mahmoud, M. A. W., Al-Nagar, H. SH. (2009): On generalized order statistics from linear exponential distribution and its characterization. Stat Papers, 50, 407-418. 
43. Nagaraja, H. N. (1988a): Record values and related statistics - A review. Communictions in Statistics - Theory and Methods, 17, 2223-2238.

44. Nagaraja, H. N. (1988b): Some characterizations of continuous distributions based on regressions of adjacent order statistics and record values. Sankhya, Series A, 50, 70-73.

45. Nelson, R. R. and Sidney, G. W (1982): An Evolutionary Theory of Economic Change, Belknap Press/Harvard University Press: Cambridge.

46. Nikulin, M., Haghighi, F. (2006): A chi-squared test for the generalized power weibull family for thehead-and-neck cancer censored data. Journal of Mathematical sciences, 133(3), 13331341.

47. Pugh, E.L. (1963): The best estimate of reliability in the exponential case. Operations Research, 11, 57-61.

48. Raqab, M. Z., Kundu, D. (2005): Comparison of different estimators of $P(Y<X)$ for a scaled Burr type $\mathrm{X}$ distribution. Communications in Statistics-Simulation and Computation, 34(2), 465-483.

49. Razmkhah, M., Ahmadi, J. (2011): Comparing two sampling schemes based on entropy of record statistics. Stat. Papers, 53, 95-106

50. Rohtagi, V. K., Saleh, A. K. Md. Ehsanes (2012): An Introduction to Probability and Statistics, Second Edition. John Wiley \& Sons, U.K.

51. Sathe, Y. S., Shah, S. P. (1981): On estimating $\mathrm{P}(\mathrm{X}<\mathrm{Y})$ for the exponential distribution. Commun. Statist. - Theor. Meth., A10, 39-47.

52. Sinha, S. K. (1986): Reliability and Life Testing. Wiley Eastern Limited, New Delhi.

53. Tadikamalla, P. R. (1980): A look at the Burr and related distributions. Inter. Statist. Rev., 48, 337-344.

54. Tong, H. (1974): A note on the estimation of $\mathrm{P}(\mathrm{Y}<\mathrm{X})$ in the exponential case. Technometrics, 16, 625.

55. Tong, H. (1975): Letter to the editor. Technometrics, 17, 393.

56. Tyagi, R. K., Bhattacharya, S. K.(1989): A note on the MVU estimation of reliability for the Maxwell failure distribution. Estadistica, 41, 73-79.

57. Watson, R. I. (1952): Research design and methodology in evaluating the results of psychotherapy, J. Clin. Psychol., 8, 29-33. DOI:10.1002/1097-4679(195201)8:1<29::AIDJCLP2270080107>3.0.CO;2-O 